\title{
American options and the free boundary exercise region: a PDE approach
}

\author{
G. RAPUCH ${ }^{\dagger}$ \\ CREST Finance-Assurance, 15, boulevard Gabriel Péri, 92245 Malakoff Cedex, France
}

[Received 22 July 2003 and in revised form 23 July 2004]

\begin{abstract}
American options are classical financial derivative contracts which lead to free boundary problems. The objective of this article is to give some qualitative properties of the exercise region of American options by means of analytic techniques. We prove that the price of an American option is the unique viscosity solution of an obstacle problem. We also prove comparison principles and strict comparison principles. These results enable us to localize the exercise region and to prove the propagation of convexity for American options. As a result, we study the influence of the volatility parameter on the price of American options.
\end{abstract}

\section{Introduction}

This article is devoted to the study of some qualitative properties of American options. Option contracts are financial instruments which have been introduced as means to hedge against certain risks. An American option allows its owner to receive a reward at any time up to the expiry date $T$. His aim is then to maximize his gain.

We will study options written on $n$ underlying assets with prices $\left(S_{t}^{1}, \ldots, S_{t}^{n}\right)$. In a generalized Black-Scholes framework [7], the logarithms of prices

$$
X_{t}=\left(\ln \left(S_{t}^{1}\right), \ldots, \ln \left(S_{t}^{n}\right)\right)
$$

solve a stochastic differential equation (SDE) under the risk-neutral probability which we assume to exist. We will not discuss here the relevance of such an assumption. As is classical, we assume that the SDE has the form

$$
\mathrm{d} X_{t}=b\left(t, X_{t}\right) \mathrm{d} t+\sigma\left(t, X_{t}\right) \mathrm{d} W_{t},
$$

where $W_{t}$ is an $m$-dimensional standard Brownian motion under the risk-neutral probability associated to the $\mathbb{P}$-completion $\left(\mathcal{F}_{t}\right)$ of its natural filtration. This kind of SDE takes into account the interest rate and dividends payed by the asset (notice that $m$ is not necessarily equal to $n$ ).

We are particularly concerned with an American option with finite expiry date $T$. When the option is exercised at time $t$, the owner receives the payoff $\psi\left(X_{t}\right)$ corresponding to the logarithms of prices $X_{t}$.

Bensoussan [5] and then Karatzas [20] first obtained a representation for the price of an American option as the supremum over $\Theta_{t, T}$ (the set of adapted stopping times in $[t, T]$ a.e.) of the expectation of the discounted payoff: the price of an American option with payoff $\psi\left(X_{t}\right)$ is given by

$$
u(t, x):=\sup _{\theta \in \Theta_{t, T}} E\left(e^{-\int_{t}^{\theta} r(s) \mathrm{d} s} \psi\left(X_{\theta}^{t, x}\right)\right),
$$

†E-mail: rapuch@ensae.fr, rapuch@noos.fr 
where $X_{s}^{t, x}$ is the solution of the SDE at time $s \geqslant t$ such that $X_{t}^{t, x}=x$. This point of view relies on the theoretical link between optimal stopping times and variational inequalities developed in [4], [5] and [20]. Furthermore, this theory states that the optimal stopping time is given by

$$
\tau^{*}=\inf \left\{s \in[t, T] \mid u\left(s, X_{s}^{t, x}\right)=\psi\left(X_{s}^{t, x}\right)\right\} .
$$

These results emphasize the key role of the exercise region

$$
\mathcal{E}=\left\{( t , x ) \in \left[0, T\left[\times \mathbb{R}^{n} \mid u(t, x)=\psi(x)\right\} .\right.\right.
$$

Indeed, $u$ is always greater than $\psi$ and the first time when $u\left(s, X_{S}\right)$ becomes equal to $\psi\left(X_{S}\right)$ is optimal to exercise the option. Moreover, we will see that $u$ solves the standard Black-Scholes PDE in the complement of $\mathcal{E}$ which is called the continuation region.

The understanding of the exercise region is thus crucial to evaluating the American option and is of obvious practical importance. This article is mainly aimed at determining this region and deriving some mathematical properties of it. The study of an American option is thus equivalent to the study of a free boundary problem. McKean [23] and van Moerbeke [29] showed the link between optimal stopping and free boundary problems. The pricing of American options leads to equations not unlike several models in physics as the one-phase Stefan problem although the smooth fit at the free boundary is not the same (see Rodrigues [27] for a review on the subject).

The obstacle problem is modelled by the following nonlinear PDE:

$$
\max \left(\psi-u, \frac{\partial u}{\partial t}+\mathcal{L} u\right)=0
$$

with the operator

$$
\mathcal{L} f=\sum_{i, j} a_{i j}(t, x) \frac{\partial^{2} f}{\partial x_{i} \partial x_{j}}+\sum_{i} b_{i}(t, x) \frac{\partial f}{\partial x_{i}}-r(t) f,
$$

where $A=\left(a_{i j}\right)=\sigma \sigma^{*} / 2$ and $r$ is the instantaneous interest rate.

We recall the equivalence between the obstacle problem and the stopping time problem in Section 2, particularly in Proposition 8 . From Section 3 on, we concentrate on the framework of the obstacle problem to study American options.

The first authors who studied the exercise region for two-asset American options were Broadie and Detemple [8]. Villeneuve [30] further characterized the nonemptiness of the exercise region and showed a criterion to localize the exercise region in more general cases. He showed that

$$
\mathcal{L} \psi \text { is a nonzero positive measure } \Leftrightarrow \mathcal{E}=\emptyset \text {. }
$$

However, these results are essentially restricted to the cases of constant volatility and constant interest rate. It is known that in most market situations, these assumptions are too restrictive. The payoff functions are also less general than in our framework.

After this work was completed, we have learned about a paper by Kampen [19], independent of our work. He studied some generalizations of Villeneuve's results [30] using viscosity solutions. He essentially investigated the shape of the exercise region near expiry and the regularity of the free boundary when the payoff is a bounded function. He also showed the continuity of the free boundary for non-time-dependent coefficients; in the case of time-dependent coefficients he needed additional regularity of the payoff function. 
Our main contribution is an extension of Villeneuve's results to a more general framework where the volatility may depend on the asset prices and on time. We also consider a more general class of payoff functions, the continuous nonnegative functions with exponential growth with respect to the logarithm of asset prices.

In this regard, it will be convenient to state that the price of an American option is a solution of a variational inequality in the viscosity sense; this concept is reviewed in Section 2 The link between optimal stopping theory and viscosity solutions for the present problem is established in Proposition 4

One of the central notions of this article is the comparison principle. We obtain different forms of such a principle for the obstacle problem, in particular in Sections 2 and 3 (Theorems 3 and 9). The main difficulty in proving these principles is the degeneracy of the operators. These principles imply many useful properties. In particular, they ensure uniqueness and we use them to compare the solution of the obstacle problem and the obstacle. With this method, we prove an extension of Villeneuve's results (Propositions 12 and 13 .

Then, in Section 4 we show how the use of the same kind of methods can lead to other qualitative properties such as the convexity of the solution or the influence of the volatility parameter on the option prices. We show that for options written on one underlying asset $(n=1)$, if the payoff is a convex function of the asset price, then so is the price of the American option (Proposition 16). In addition, for such options, we study the influence of the volatility parameter on the price by means of comparison principles. Here we extend the results of Touzi [28] to more general options. The main result is that the price of an option increases when the volatility parameter increases (Proposition 17).

Finally, in Section 5 we study further options such as the American call on the maximum of two underlying assets.

\section{Assumptions and notations}

We consider American options written on $n$ underlying assets with prices $\left(S_{t}^{1}, \ldots, S_{t}^{n}\right)$. In a generalized Black-Scholes setting, the logarithms of prices $X_{t}=\left(\ln \left(S_{t}^{1}\right), \ldots, \ln \left(S_{t}^{n}\right)\right)$ solve a stochastic differential equation under the risk-neutral probability:

$$
\mathrm{d} X_{t}=b\left(t, X_{t}\right) \mathrm{d} t+\sigma\left(t, X_{t}\right) \mathrm{d} W_{t},
$$

where $W_{t}$ is a standard Brownian motion under the risk-neutral probability associated to the $\mathbb{P}$ completion $\left(\mathcal{F}_{t}\right)$ of its natural filtration. If the option is exercised at time $t$, the owner receives a payoff $\psi\left(X_{t}\right)$.

We will assume that the following hypotheses hold:

(H1) $\quad b$ is a bounded function from $[0, T] \times \mathbb{R}^{n}$ into $\mathbb{R}^{n}$, and $\sigma$ is a bounded function from $[0, T] \times \mathbb{R}^{n}$ into $M_{n, m}(\mathbb{R})$. Furthermore, there exists $K>0$ such that for all $x, y$ in $\mathbb{R}^{n}$ and $s, t \in[0, T]$,

$$
\|\sigma(t, x)-\sigma(s, y)\|+\|b(t, x)-b(s, y)\| \leqslant K(\|x-y\|+|s-t|) .
$$

(H2) Uniform ellipticity: The matrix $A=\left(a_{i j}\right)=\sigma \sigma^{*} / 2$ is coercive, i.e.,

$$
\exists \eta>0 \forall(t, x) \in[0, T] \times \mathbb{R}^{n} \forall \xi \in \mathbb{R}^{n}, \quad \sum_{i, j} a_{i j}(t, x) \xi_{i} \xi_{j} \geqslant \eta|\xi|^{2} .
$$


(H3) The interest rate $t \mapsto r(t)$ is positive and continuous.

(H4) $\psi$ is a continuous nonnegative function such that

$$
\exists M>0, \quad \psi(x) \leqslant M e^{M\|x\|} .
$$

(H1) ensures existence and uniqueness of the solution of the SDE. In addition, these hypotheses will guarantee the validity of the maximum principles. (H2) is essential to demonstrating the strict comparison principle whereas the maximum principles only require ellipticity and not uniform ellipticity.

Let

$$
u(t, x):=\sup _{\theta \in \Theta_{t, T}} E\left(e^{-\int_{t}^{\theta} r(s) \mathrm{d} s} \psi\left(X_{\theta}^{t, x}\right)\right) ;
$$

the optimal stopping theory (see e.g. [12]) asserts that

$$
u\left(t, X_{t}\right)=\underset{\theta \in \Theta_{t, T}}{\operatorname{ess} \sup } E\left(e^{-\int_{t}^{\theta} r(s) \mathrm{d} s} \psi\left(X_{\theta}^{t, x}\right) \mid \mathcal{F}_{t}\right)
$$

is the value of the American option with payoff $\psi$. Furthermore, this theory yields the optimal stopping time in $\Theta_{t, T}$, the set of all $\left(\mathcal{F}_{t}\right)$-adapted stopping times whose values are in $[t, T]$ a.e. This time $\tau^{*}$ is defined by

$$
\tau^{*}=\inf \left\{s \in[t, T] \mid u\left(s, X_{s}^{t, x}\right)=\psi\left(X_{s}^{t, x}\right)\right\} .
$$

A straightforward adaptation of [18] to the multivariate case yields the continuity of the price option under the present hypotheses.

PROPOSITION $1 \quad u$ is a continuous function in $C^{0}\left([0, T] \times \mathbb{R}^{n}\right)$.

\section{The obstacle problem and viscosity solutions}

Jaillet, Lamberton and Lapeyre [18] showed that the price of an American option is a solution of a variational inequality, which can be written, when all the functions are smooth, as

$$
\max \left(\psi-u, \frac{\partial u}{\partial t}+\mathcal{L}^{t} u\right)=0
$$

where $\mathcal{L}^{t}$ denotes the operator

$$
\mathcal{L}^{t} f=\sum_{i, j} a_{i j}(t, x) \frac{\partial^{2} f}{\partial x_{i} \partial x_{j}}+\sum_{i} b_{i}(t, x) \frac{\partial f}{\partial x_{i}}-r(t) f .
$$

We denote $\mathcal{L}^{t}$ simply by $\mathcal{L}$ if its coefficients do not depend on time.

In order to study this equation in the most general case, we refer to the concept of viscosity solutions. After the basic definitions, we show that a comparison principle holds for the obstacle problem and, finally, that its unique solution is given by a solution of the stopping time problem. In other words, the solution of the obstacle problem with obstacle function $\psi$ is the price of the American option with payoff $\psi$.

Let $C^{1,2}\left([0, T] \times \mathbb{R}^{n}\right)$ be the set of all functions twice continuously differentiable in $x$ and once in $t$. A function in $C^{1,2}\left([0, T] \times \mathbb{R}^{n}\right)$ will be called a test function. Let us recall the definition of viscosity solutions (see [11] for more details). 
DEFINITION 1 Let $\Omega$ be an open subset of $\mathbb{R}^{n}$. Let $F$ be a continuous function from $\mathbb{R} \times \mathbb{R}^{n} \times$ $\mathbb{R} \times \mathbb{R}^{n} \times M_{n}(\mathbb{R}) \times \mathbb{R}$ into $\mathbb{R}$, nondecreasing with respect to the $M_{n}(\mathbb{R})$ variable. Let $\theta$ and $\chi$ be two continuous functions from $[0, T] \times \mathbb{R}^{n}$ into $\mathbb{R}$. A function $v$ upper semicontinuous (u.s.c.) on $\Omega \times[0, T]$ and defined on $\bar{\Omega} \times[0, T]$ is said to be a viscosity subsolution on $\Omega \times[0, T]$ of

$$
\left\{\begin{array}{l}
F\left(t, x, u, D_{x} u, D_{x x} u, u_{t}\right)=0, \\
\forall x \in \Omega, \quad u(T, x)=\theta(T, x) \\
\forall x \in \partial \Omega, t \in[0, T[, \quad u(t, x)=\chi(t, x),
\end{array}\right.
$$

if for any $\phi \in C^{1,2}(\Omega \times] 0, T[)$ such that $v-\phi$ has a local maximum at $\left(t_{0}, x_{0}\right)$ with $v\left(t_{0}, x_{0}\right)=$ $\phi\left(t_{0}, x_{0}\right)$ we have

$$
\left\{\begin{array}{l}
F\left(t_{0}, x_{0}, \phi\left(t_{0}, x_{0}\right), \phi_{x}\left(t_{0}, x_{0}\right), \phi_{x x}\left(t_{0}, x_{0}\right), \phi_{t}\left(t_{0}, x_{0}\right)\right) \geqslant 0 \\
\forall x \in \partial \Omega, t \in[0, T[\quad v(t, x) \leqslant \theta(t, x) \\
\forall x \in \Omega, \quad v(T, x) \leqslant \chi(T, x)
\end{array}\right.
$$

$v$ is a supersolution if $v$ is a lower semicontinuous (l.s.c.) function and if the opposite inequalities are satisfied whenever $v-\phi$ has a local minimum.

A viscosity solution is a continuous function which is both a subsolution and a supersolution.

REMARK 1 As mentioned in [3] or [11], the definition of viscosity solutions can be equivalently stated with local minima or maxima replaced with global minima or maxima. We can also consider only strict extrema.

We will show in Proposition 4 that the prices of American options with payoff $\psi$ solve (S) in the viscosity sense, where

$$
\left\{\begin{array}{l}
\max \left(\psi-u, \partial u / \partial t+\mathcal{L}^{t} u\right)=0 \quad \text { on }\left[0, T\left[\times \mathbb{R}^{n}\right.\right. \\
\forall x \in \mathbb{R}^{n}, \quad u(T, x)=\psi(x)
\end{array}\right.
$$

Here, we have

$$
F\left(t, x, u, D_{x} u, D_{x x} u, u_{t}\right)=\max \left(\psi(x)-u(t, x), \frac{\partial u}{\partial t}(t, x)+\mathcal{L}^{t} u(t, x)\right) .
$$

Under our reasonable hypotheses we demonstrate the uniqueness of solutions for (S) (Proposition 4 states that the unique solution is the price of an American option with payoff $\psi$ ). First, we demonstrate a comparison principle on a bounded domain. This result will be used to demonstrate the comparison principle on the whole space $\mathbb{R}^{n}$. We use the methods of [10] and [11] for parabolic PDEs. This result was shown by Villeneuve [31] in the case of constant coefficients in a onedimensional case.

Proposition 2 Let $\left(\mathrm{S}_{0}\right)$ be the following system of equations:

$$
\left\{\begin{array}{l}
\max \left(\psi-u, \partial u / \partial t+\mathcal{L}^{t} u\right)=0 \quad \text { on }[0, T[\times \Omega, \\
\forall x \in \Omega, \quad u(T, x)=\theta(T, x) \\
\forall x \in \partial \Omega, t \in[0, T[, \quad u(t, x)=\theta(t, x)
\end{array}\right.
$$

with

$$
\mathcal{L}^{t} f=\sum_{i, j} a_{i j}(t, x) \frac{\partial^{2} f}{\partial x_{i} \partial x_{j}}+\sum_{i} b_{i}(t, x) \frac{\partial f}{\partial x_{i}}-c(t, x) u,
$$

where $c>0$ and $a_{i j}, b_{i}, c, \psi, \theta$ are continuous and where the $b_{i} \mathrm{~s}$ are locally Lipschitz. If $\Omega$ is a 
bounded domain, $u$ (resp. $v$ ) a viscosity subsolution (resp. a supersolution) of $\left(\mathrm{S}_{0}\right)$, and $v \geqslant u$ on the parabolic boundary $\partial \Omega_{p}$ of $\Omega$, i.e.

$$
\left\{\begin{array}{l}
\forall x \in \Omega, \quad v(T, x) \geqslant u(T, x) \\
\forall x \in \partial \Omega, t \in[0, T[, \quad v(t, x) \geqslant u(t, x)
\end{array}\right.
$$

then $v \geqslant u$ on $\Omega \times[0, T]$.

Proof. The proof is by standard arguments. See [3] or [22] for more details.

REMARK 2 In fact, we only used the nonnegativity of $A$. The proof is the same if we only assume $\mathcal{L}^{t}$ to be degenerate elliptic. We can prove a comparison principle for a viscosity elliptic degenerate equation similar to the previous one, in particular for a parabolic equation (with condition on the elliptic boundary). We will use this remark several times to demonstrate the strict comparison principles in Section 3.2

We next extend the comparison principle on $\mathbb{R}^{n}$ for the system $(\mathrm{S})$. This was shown in [12] when the functions grow at most polynomially. Here we study the more general case of exponential growth which allows more general payoff functions (e.g. the American call).

THEOREM 3 Assume that the coefficients of $\mathcal{L}^{t}$ are bounded and locally Lipschitz, and that $\psi$ is a continuous function. Let $u$ (resp. $v$ ) be a subsolution (resp. a supersolution) of

$$
\left\{\begin{array}{l}
\max \left(\psi-w, \partial w / \partial t+\mathcal{L}^{t} w\right)=0 \\
\forall x \in \mathbb{R}^{n}, \quad w(T, x)=\psi(x)
\end{array}\right.
$$

such that $u(T, x) \leqslant v(T, x)$ for all $x \in \mathbb{R}^{n}$. If in addition there exist positive constants $C, \lambda$ such that for all $(t, x) \in[0, T] \times \mathbb{R}^{n}$,

$$
|u(t, x)| \leqslant C e^{\lambda|x|}, \quad|v(t, x)| \leqslant C e^{\lambda|x|}, \quad|\psi(x)| \leqslant C e^{\lambda|x|},
$$

then $u(t, x) \leqslant v(t, x)$ for all $(t, x)$.

Proof. We use a change of function in order to use the result on bounded sets (see e.g. [6]). Given a function $f$, we define

$$
\bar{f}:=\frac{e^{\alpha t} f(t, x)}{\operatorname{ch}\left(\beta x_{1}\right) \cdots \operatorname{ch}\left(\beta x_{n}\right)},
$$

where ch stands for the hyperbolic cosine, i.e. $\operatorname{ch}(u)=\left(e^{u}+e^{-u}\right) / 2$ for $u \in \mathbb{R}$. In this proof we also use the notation th $(u)=\left(e^{u}-e^{-u}\right) /\left(e^{u}+e^{-u}\right)$. Thus, a simple calculation yields

$$
\mathcal{L}^{t} f=\left(e^{-\alpha t} \operatorname{ch}\left(\beta x_{1}\right) \cdots \operatorname{ch}\left(\beta x_{n}\right)\right)\left[\bar{f}_{t}+a_{i j} \frac{\partial^{2} \bar{f}}{\partial x_{i} \partial x_{j}}+\bar{b}_{i} \frac{\partial \bar{f}}{\partial x_{i}}-\bar{c} \bar{f}\right]=\left(\operatorname{ch}\left(\beta x_{1}\right) \cdots \operatorname{ch}\left(\beta x_{n}\right)\right) \overline{\mathcal{L}^{t}} \bar{f}
$$

with

$$
\begin{aligned}
\bar{b}_{i} & =b_{i}+\sum_{j} 2 \beta \operatorname{th}\left(\beta x_{j}\right) a_{i j} \quad \forall i \\
\bar{c} & =-\sum_{i \neq j} a_{i j} \beta^{2} \operatorname{th}\left(\beta x_{i}\right) \operatorname{th}\left(\beta x_{j}\right)-\sum_{i}\left(b_{i} \beta \operatorname{th}\left(\beta x_{i}\right)+\beta^{2} a_{i i}\right)+c+\alpha .
\end{aligned}
$$

We then choose $\alpha>\sum_{i, j}\left\|a_{i j}\right\|_{\infty} \beta^{2}+\sum_{i}\left\|b_{i}\right\|_{\infty} \beta$ such that $\bar{c}>0$ and $\beta>\lambda$. 
Let $\bar{u}$ and $\bar{v}$ be a viscosity subsolution and supersolution of

$$
\left\{\begin{array}{l}
\max (\bar{\psi}-\bar{u}, \partial \bar{u} / \partial t+\overline{\mathcal{L}} \bar{u})=0, \\
\forall x \in \mathbb{R}^{n}, \quad \bar{u}(T, x)=\bar{\psi}(T, x) .
\end{array}\right.
$$

Then uniformly in $t$,

$$
\lim _{|x| \rightarrow \infty} \bar{u}(t, x)=\lim _{|x| \rightarrow \infty} \bar{v}(t, x)=0 .
$$

So, for every $\delta>0$ there is $R_{\delta} \geqslant 1 / \delta$ such that for all $x \in \partial B_{R_{\delta}}$ and $\left.t \in\right] 0, T$,

$$
\bar{u}(t, x) \leqslant \bar{\psi}+\delta / 2 \leqslant \bar{v}(t, x)+\delta .
$$

Let us examine the following system with respect to $g$ :

$$
\left\{\begin{array}{l}
\max (\bar{\psi}-g, \partial g / \partial t+\overline{\mathcal{L}} g)=0, \\
\forall x \in B_{R_{\delta}}, \quad g(T, x)=\psi(T, x)+\delta / 2, \\
\forall x \in \partial B_{R_{\delta}}, \quad g(t, x)=\psi(t, x)+\delta / 2 .
\end{array}\right.
$$

Then $\bar{v}+\delta$ is a supersolution of $\left(\mathrm{S}_{\delta}\right)$ and $\bar{u}$ is a subsolution. We can use the comparison principle for the bounded open set $B_{R_{\delta}}$ :

$$
\forall(t, x) \in B_{R_{\delta}} \times[0, T], \quad \bar{u}(t, x) \leqslant \bar{v}+\delta .
$$

We fix $(t, x)$ and let $\delta$ tend to 0 to get $u(t, x) \leqslant v(t, x)$.

In particular, we can conclude that there is at most one viscosity solution of

$$
\left\{\begin{array}{l}
\max \left(\psi-w, \partial w / \partial t+\mathcal{L}^{t} w\right)=0, \\
\forall x \in \mathbb{R}^{n}, \quad w(T, x)=\psi(T, x) .
\end{array}\right.
$$

REMARK 3 In both the viscosity solution and the optimal stopping context we assumed that $\psi$ is continuous and $|\psi(x)| \leqslant C e^{\lambda|x|}$. It is no more restrictive to assume $|u| \leqslant C e^{\lambda|x|}$. Indeed, in the optimal stopping viewpoint the price of the American option is

$$
u(t, x)=\sup _{\theta \in \Theta_{t, T}} E\left(e^{-\int_{t}^{\theta} r(s) \mathrm{d} s} \psi\left(X_{\theta}^{t, x}\right)\right) .
$$

So if $|\psi(x)| \leqslant C e^{\lambda|x|}$, then using the inequality

$$
\forall M>0 \exists C>0 \quad E\left(\sup _{0 \leqslant t \leqslant T} e^{M\left|X_{t}^{0, x}\right|}\right) \leqslant C e^{M|x|}
$$

one gets

$$
|u(t, x)| \leqslant C^{\prime} e^{\lambda|x|} .
$$

Finally, we state the link between optimal stopping theory and viscosity solutions. We recall that $\Theta_{t, T}$ is the set of all $\left(\mathcal{F}_{t}\right)$-adapted stopping times whose values are in $[t, T]$ a.s. 
Proposition 4 If $\psi$ is continuous and such that $|\psi(x)| \leqslant C e^{\lambda|x|}$ (that is, (H4) is satisfied), then the function defined by

$$
u(t, x)=\sup _{\theta \in \Theta_{t, T}} E\left(e^{-\int_{t}^{\theta} r(s) \mathrm{d} s} \psi\left(X_{\theta}^{t, x}\right)\right)
$$

is a viscosity solution of

$$
\left\{\begin{array}{l}
\max \left(\psi-u, \partial u / \partial t+\mathcal{L}^{t} u\right)=0 \\
\forall x \in \mathbb{R}^{n}, \quad u(T, x)=\psi(x) .
\end{array}\right.
$$

Moreover, $u$ is the unique solution such that there exist $C^{\prime}>0$ and $\lambda>0$ such that $|u(t, x)| \leqslant$ $C^{\prime} e^{\lambda|x|}$.

Proof. The argument is standard and can be found in [22].

The comparison principle enables us to recover some well known qualitative results. Indeed, the European option is clearly a subsolution of (S). Therefore the American option is more expensive than the European one.

Proposition 5 (1) $u(t, x) \geqslant \psi(x)$ for all $(t, x) \in[0, T] \times \mathbb{R}^{n}$.

(2) If $\sigma, r$ and $b$ do not depend on time (we say that the diffusion is homogeneous) then $u(\cdot, x)$ is nonincreasing for each $x \in \mathbb{R}^{n}$.

Proof. (1) is true since $\psi$ is a subsolution of (S). To prove (2) we use the comparison principle again, letting $v(t, x)=u(t-h, x)$ for $h>0$ and $t \in[h, T]$. We have $v(T, x)=u(T-h, x) \geqslant \psi(x)$. So $v$ is a subsolution of

$$
\left\{\begin{array}{l}
\max \left(\psi-u, \partial u / \partial t+\mathcal{L}^{t} u\right)=0 \\
\forall x \in \mathbb{R}^{n}, \quad u(T, x)=\psi(T, x)
\end{array}\right.
$$

and $u(t-h, x) \geqslant u(t, x)$.

REMARK 4 The price of an European option is more expensive than its payoff iff the corresponding American option coincides with the European option.

This remark can be used to derive directly Villeneuve's results [30] characterizing the American options which are in fact European options. The advantage is that we can allow here the diffusion to be homogeneous and not only constant to deduce that the class of such options is the options whose payoff $\psi$ is such that $\mathcal{L} \psi$ is a nonzero positive measure. However, the results of the next section are even more general.

\section{Comparison principles and the exercise region}

\subsection{Definition and first properties}

The goal of this section is to study the exercise region

$$
\mathcal{E}=\left\{( t , x ) \in \left[0, T\left[\times \mathbb{R}^{n} \mid u(t, x)=\psi(x)\right\} .\right.\right.
$$

Such a denomination has a financial interpretation. Whenever the price of the option is larger than the payoff, it is more profitable to sell the option than exercise it. Thus, it is not reasonable to exercise the option outside of the exercise region.

On the other hand, the first time this area is reached, it is optimal to exercise the option (according to the stopping time theory). 
In order to understand the behavior of American options, it is crucial to determine this region. On the one hand, $\psi$ is known (thus $u$ is known in $\mathcal{E}$ ) and on the other hand $u$ solves the standard BlackScholes PDE in the complement of $\mathcal{E}$, which is called the continuation region. When the region $\mathcal{E}$ is empty, it is never optimal to exercise the option before expiry; the option is then a European option. This is the case for an American call written on a non-dividend-paying asset (Merton's result [24]).

The next result was shown by Villeneuve [30] in the case of constant coefficients. Using the comparison principle, we extend it to a general diffusion.

PROPOSITION 6 The exercise region of an American option with bounded payoff is nonempty.

Proof. If the exercise region were empty, the price $u$ of the option would solve

$$
\frac{\partial u}{\partial t}+\mathcal{L}^{t} u=0
$$

with final condition $u(T, x)=\psi(x)$. The function $w(t, x)=\exp \left(-\int_{t}^{T} r(s) \mathrm{d} s\right)\|\psi\|_{\infty}$ is a supersolution of the last equation. So the comparison principle implies

$$
u(t, x) \leqslant w(t, x),
$$

and as $u \geqslant \psi$, we would get

$$
\psi(x) \leqslant \exp \left(-\int_{t}^{T} r(s) \mathrm{d} s\right)\|\psi\|_{\infty}
$$

which is false.

REMARK 5 If $\psi$ reaches its supremum at $x_{0}$, then it is optimum to exercise the option at any time when the price is $x_{0}$. Thus, $\psi\left(x_{0}\right)=u\left(t, x_{0}\right)$. For example, the American put with payoff $\psi(x)=(K-x)_{+}$has a nonempty exercise region and $u(t, 0)=K$ for all $t$.

We want to exhibit some criteria to localize the exercise region. If $\mathcal{L}^{t}$ is the diffusion operator

$$
\mathcal{L}^{t} f=\sum_{i, j} a_{i j}(t, x) \frac{\partial^{2} f}{\partial x_{i} \partial x_{j}}+\sum_{i} b_{i}(t, x) \frac{\partial f}{\partial x_{i}}-r(t) f
$$

a necessary and sufficient condition for the emptiness of the exercise region when $a_{i j}, b_{i}, r$ are constant is given by Villeneuve [30]:

$$
\mathcal{L} \psi \text { is a nonzero positive measure } \Leftrightarrow \mathcal{E}=\emptyset .
$$

We give an extension of this result:

Proposition 7 Assume that, for all $t \in\left[0, T\left[, \mathcal{L}^{t} \psi\right.\right.$ is positive in the viscosity sense but differs from 0 on an open connected subset $U$ (i.e. $\psi$ is a strict viscosity subsolution of $\mathcal{L}^{t} w=0$ ). Then

$$
\forall(t, x) \in[0, T[\times U, \quad u(t, x)>\psi(x) .
$$

That is to say,

$$
\mathcal{E} \cap([0, T[\times U)=\emptyset .
$$

To achieve this objective, we show a strict comparison principle between viscosity subsolutions and supersolutions for a linear problem. This principle will give a strict comparison between the price $u$ of an American option and its payoff $\psi$. Such a comparison means that we are in the continuation region. Nirenberg [25] first showed a strict comparison principle for classical parabolic 
equations. Bardi and Da Lio [2] showed a strict maximum principle (i.e. a comparison between a supersolution and 0 ) in the viscosity context for parabolic nonlinear equations. However, the nonlinearity of viscosity solutions prevents us from using these results to get a comparison principle between a supersolution and a subsolution.

Following [25] and [2], we first show a propagation at a given time, then a propagation in the space variables. In other words, we first show that a supersolution $v$ and a subsolution $u$ are such that $v>u$ or $v=u$ by proving it first on the lines $t=$ const.

\subsection{Strict comparison principle for a linear problem in the viscosity sense}

Proposition 8 Assume that the coefficients of $\mathcal{L}^{t}$ are bounded and locally Lipschitz, and that $\psi$ is a continuous function. Let $U$ be a domain in $\mathbb{R}^{n}$. Let $u$ (resp. $v$ ) be a viscosity subsolution (resp. a viscosity supersolution) of

$$
\left\{\begin{array}{l}
\partial w / \partial t+\mathcal{L}^{t} w=0 \\
\forall x \in U, \quad w(T, x)=\psi(T, x)
\end{array}\right.
$$

such that $u(t, x) \leqslant v(t, x)$ for all $t \in[0, T]$ and $x \in U$. If there exist $\left.t_{0} \in\right] 0, T\left[\right.$ and $x_{0} \in U$ such that $u\left(t_{0}, x_{0}\right)=v\left(t_{0}, x_{0}\right)$, then $u\left(t_{0}, x\right)=v\left(t_{0}, x\right)$ for all $x \in U$.

Proof. We first prove that if there exists a closed ball $B$ with radius $R$ and center $P=\left(t_{1}, x_{1}\right)$ included in ]0,T[ $\times U$ such that $u<v$ in the interior of $B$ and there is a point $P_{2}=\left(t_{2}, x_{2}\right)$ belonging to the boundary of $B$ such that $u\left(P_{2}\right)=v\left(P_{2}\right)$, then $x_{2}=x_{1}$. Indeed, suppose that $x_{2} \neq x_{1}$; we can also assume that $P_{2}$ is the only point such that $u=v$ in $B$ (or else we can change the center and decrease the radius of the ball). We then introduce a ball with center $P_{2}$ included in ]0, $T$ [ $\times U$ whose radius is $r<\left\|x_{1}-x_{2}\right\|$. We also introduce an auxiliary function

$$
\Phi(t, x):=e^{-\gamma\left(\left\|x-x_{1}\right\|^{2}+\left|t-t_{1}\right|^{2}\right)}-e^{-\gamma R^{2}} .
$$

We notice that $\Phi \in C^{\infty}, \Phi>0$ in $B, \Phi=0$ on $\partial B$ and $\Phi<0$ outside of $B$. Let

$$
\alpha(t, x)=e^{-\gamma\left(\left\|x-x_{1}\right\|^{2}+\left|t-t_{1}\right|^{2}\right)} .
$$

A simple calculation yields

$$
\begin{aligned}
\Phi_{t}(t, x) & =-2 \gamma\left(t-t_{1}\right) \alpha(t, x), \\
\Phi_{x_{i}}(t, x) & =-2 \gamma\left(x_{i}-x_{1, i}\right) \alpha(t, x), \\
\Phi_{x_{i} x_{j}}(t, x) & =-2 \gamma \delta_{i j} \alpha(t, x)+4 \gamma^{2}\left(x_{i}-x_{1, i}\right)\left(x_{j}-x_{1, j}\right) \alpha .
\end{aligned}
$$

Therefore,

$$
\begin{aligned}
\frac{\partial \Phi}{\partial t}+\mathcal{L}^{t} \phi(t, x)= & -2 \gamma \sum a_{i i} \alpha+4 \gamma^{2} \sum a_{i j}\left(x_{i}-x_{1, i}\right)\left(x_{j}-x_{1, j}\right) \alpha \\
& +\sum b_{i}\left(-2 \gamma\left(x_{i}-x_{1, i}\right) \alpha(t, x)\right)-r \Phi
\end{aligned}
$$

According to the ellipticity hypothesis (H2), we have

$$
4 \gamma^{2} \sum a_{i j}\left(x_{i}-x_{1, i}\right)\left(x_{j}-x_{1, j}\right) \alpha \geqslant \eta 4 \gamma^{2}\left\|x-x_{1}\right\|^{2} .
$$


Thus, as $\left\|x_{2}-x_{1}\right\|$ is positive, there exists $\gamma$ large enough such that

$$
\left(\frac{\partial \Phi}{\partial t}+\mathcal{L}^{t} \phi\right)\left(t_{2}, x_{2}\right)>0
$$

Then, $\Phi$ being smooth, there exists a ball $B\left(P_{2}, r_{0}\right)$ with $r_{0}<R$ such that $\partial \Phi / \partial t+\mathcal{L}^{t} \phi>0$ on $B\left(P_{2}, r_{0}\right)$. Let $X:=B\left(P_{2}, r_{0}\right) \cap B(P, R)$. On $\partial X$, we have $u<v$ and $\Phi>0$ on $\partial B\left(P_{2}, r_{0}\right)$, and $u \leqslant v$ and $\Phi=0$ on $\partial B(P, R)$. So, by semicontinuity, there exists $\epsilon>0$ such that $u+\epsilon \Phi \leqslant v$ on $\partial X$. As $\Phi \in C^{\infty}$, it follows that $u+\epsilon \Phi$ is a viscosity subsolution of $\partial w / \partial t+\mathcal{L}^{t} w=0$ on $X$. It suffices to notice that the comparison principle is valid for linear degenerate elliptic equations such as $w_{t}+\mathcal{L}^{t} w=0$ on $X$ (see Remark 2). We conclude that $u+\epsilon \Phi \leqslant v$ on $\bar{X}$. Indeed, this is true on $B\left(P_{2}, r_{0}\right)$ because $u \leqslant v$ and $\Phi<0$ outside of $B\left(\left(t_{1}, x_{1}\right), R\right)$. Thus $P_{2}$ is a point of local maximum for $u-v-\epsilon \Phi$ on $B\left(P_{2}, r_{0}\right)$ (which will be denoted by $B$ from now on), with $u\left(P_{2}\right)+\epsilon \Phi\left(P_{2}\right)=v\left(P_{2}\right)$. We can assume that this maximum is strict, otherwise we replace $v$ with $v+\left\|x_{2}-x\right\|^{4}+\left|t_{2}-t\right|^{4}$, which is still a viscosity supersolution at $P_{2}$.

We would like to differentiate the functions at $P_{2}$ and derive a contradiction (this method often used to establish strict comparison principle is called the Hopf lemma; see Nirenberg [25]). To circumvent the lack of regularity, we use a variable doubling procedure. Let

$$
\begin{aligned}
\phi_{\alpha}(t, x, y) & =u(t, x)+\epsilon \Phi(t, x)-v(t, y)-\frac{1}{2 \alpha}\|x-y\|^{2} \quad \text { with } \epsilon>0, \\
w(t, x) & =u(t, x)+\epsilon \Phi(t, x) .
\end{aligned}
$$

Let

$$
M_{\alpha}=\sup _{(t, x),(t, y) \in \bar{B}} \phi_{\alpha}(t, x, y) .
$$

This quantity is finite and reached at some $\left(t_{\alpha}, x_{\alpha}, y_{\alpha}\right)$ (by a compactness argument). Using compactness, we can assume that $\left(t_{\alpha}, x_{\alpha}, y_{\alpha}\right) \rightarrow(\bar{t}, \bar{x}, \bar{y})$ as $\alpha \rightarrow 0$ with $(\bar{t}, \bar{x}),(\bar{t}, \bar{y}) \in \bar{B}$. Moreover, for all $(t, x) \in \bar{B}$ we have

$$
\phi_{\alpha}(t, x, x) \leqslant M_{\alpha},
$$

which implies, on taking the supremum, that $M_{\alpha} \geqslant 0$. Thus

$$
\left\|x_{\alpha}-y_{\alpha}\right\|^{2} \leqslant 2 \alpha\left(w\left(t_{\alpha}, x_{\alpha}\right)-v\left(t_{\alpha}, y_{\alpha}\right)\right) .
$$

Now, $w$ and $-v$ are u.s.c. and thus bounded above on $\bar{B}$. In addition,

$$
M_{\alpha} \leqslant w\left(t_{\alpha}, x_{\alpha}\right)-v\left(t_{\alpha}, y_{\alpha}\right)
$$

so passing to the limit yields $\bar{x}=\bar{y}$ and

$$
\lim \sup M_{\alpha} \leqslant \lim \sup \left(w\left(t_{\alpha}, x_{\alpha}\right)-v\left(t_{\alpha}, y_{\alpha}\right)\right)=w(\bar{t}, \bar{x})-v(\bar{t}, \bar{x}) \leqslant 0 .
$$

Thus, we can assert that $M_{\alpha}$ tends to 0 as $\alpha$ tends to 0 , and

$$
0 \leqslant w\left(t_{\alpha}, x_{\alpha}\right)-v\left(t_{\alpha}, y_{\alpha}\right)
$$

because $M_{\alpha} \geqslant 0$. Thus, $0 \leqslant \lim \sup \left(w\left(t_{\alpha}, x_{\alpha}\right)-v\left(t_{\alpha}, y_{\alpha}\right)\right) \leqslant 0$. Therefore

$$
w(\bar{t}, \bar{x})-v(\bar{t}, \bar{x})=0
$$

and $\lim \frac{1}{2 \alpha}\left\|x_{\alpha}-y_{\alpha}\right\|^{2}=0$. The maximum of $w-v$ being strict on $\bar{B}$, we conclude that $(\bar{t}, \bar{x})=P_{2}$. In particular, for $\alpha$ small enough, $\left(t_{\alpha}, x_{\alpha}\right) \in B$ and $\left(t_{\alpha}, y_{\alpha}\right) \in B$. 
Using Theorem 9 in [10] once again applied to $\phi_{\alpha}$ at $\left(t_{\alpha}, x_{\alpha}, y_{\alpha}\right)$, and the viscosity properties of $u$ and $v$, we deduce that for every $\alpha>0$ there are $X, Y \in S^{n}$ and $\xi, \eta \in \mathbb{R}$ such that

$$
\left\{\begin{array}{l}
\xi+\eta=0 \\
X+Y \leqslant 0
\end{array}\right.
$$

and

$$
\left\{\begin{aligned}
\xi- & \epsilon \frac{\partial \Phi}{\partial t}\left(t_{\alpha}, x_{\alpha}\right)+\operatorname{tr}(A X)-\epsilon \operatorname{tr}\left(A D_{x}^{2} \Phi\left(t_{\alpha}, x_{\alpha}\right)\right) \\
& +\vec{b}\left(t_{\alpha}, x_{\alpha}\right) \cdot\left(\frac{x_{\alpha}-y_{\alpha}}{\alpha}\right)\left(-\epsilon \frac{\partial \Phi}{\partial t}\left(t_{\alpha}, x_{\alpha}\right)\right)-r\left(t_{\alpha}, x_{\alpha}\right) u\left(t_{\alpha}, x_{\alpha}\right) \geqslant 0, \\
-\eta & -\operatorname{tr}(A Y)+\vec{b}\left(t_{\alpha}, y_{\alpha}\right) \cdot\left(\frac{x_{\alpha}-y_{\alpha}}{\alpha}\right)-r\left(t_{\alpha}, y_{\alpha}\right) v\left(t_{\alpha}, y_{\alpha}\right) \leqslant 0 .
\end{aligned}\right.
$$

Subtracting the two inequalities, using $\operatorname{tr}(A(X+Y)) \leqslant 0$ and taking the limsup as $\alpha \rightarrow 0$, we obtain

$$
\lim \sup \left[r\left(t_{\alpha}, y_{\alpha}\right) v\left(t_{\alpha}, y_{\alpha}\right)-r\left(t_{\alpha}, x_{\alpha}\right) u\left(t_{\alpha}, x_{\alpha}\right)\right]=r\left(P_{2}\right)\left(\epsilon \Phi\left(P_{2}\right)\right) \text {. }
$$

Furthermore, the coefficients of the operator are continuous, $\Phi \in C^{\infty}, \vec{b}$ is Lipschitz and $\frac{1}{2 \alpha}\left\|x_{\alpha}-y_{\alpha}\right\|^{2} \rightarrow 0$, thus

$$
\vec{b}\left(t_{\alpha}, x_{\alpha}\right) \cdot\left(\frac{x_{\alpha}-y_{\alpha}}{\alpha}\right)-\vec{b}\left(t_{\alpha}, y_{\alpha}\right) \cdot\left(\frac{x_{\alpha}-y_{\alpha}}{\alpha}\right) \rightarrow 0
$$

We finally get

$$
-\epsilon \mathcal{L}^{t} \Phi\left(P_{2}\right) \geqslant 0 .
$$

This contradicts $\mathcal{L}^{t} \Phi\left(P_{2}\right)>0$, and so necessarily $x_{1}=x_{2}$.

We could have proved this result for an ellipsoid which is an affine image of a ball: if $u=v$ at some point of the boundary of an ellipsoid whereas $u<v$ in its interior, then the space coordinate of this point is the same as the one of the center of the ellipsoid.

We can then conclude as in Nirenberg [25], assuming that there exists $P_{0}=\left(t_{0}, x_{0}\right)$ with $t_{0} \in$ ]0, $T$ [ such that $u\left(t_{0}, x_{0}\right)=v\left(t_{0}, x_{0}\right)$ without $u-v$ being constant on $t=t_{0}$ (that is to say, that $u<v$ at some point $\left.Q=\left(t_{0}, x^{*}\right)\right)$. On a path between these two points, with $t$ constant, there exists, by semicontinuity, a first point $P_{1}$ such that $u\left(P_{1}\right)=v\left(P_{1}\right)$. Then, on the path $\left[Q, P_{1}\right.$ [, we have $u<v$. We choose a point $P=\left(t_{0}, x_{1}\right)$ on this path such that the ball with center $P$ and radius $d\left(P, P_{1}\right)$ is included in $] 0, T[\times U$. By semicontinuity, there exists a neighborhood of $P$ in which $u<v$, and then a segment $\Delta$ with center $P$ perpendicular to the $t=t_{0}$ plane, with half length $l<d\left(P, P_{1}\right)$, such that $u<v$ on it. We consider the family of ellipsoids centered at $P$ obtained by a revolution around the axis $\Delta$ and whose projection onto the plane $t=t_{0}$ is a sphere centered at $P$ and with radius $\beta$. We then let $\beta$ vary from 0 to $d\left(P, P_{1}\right)$. For $\beta=0$ it corresponds to $\Delta$ on which $u<v$. By semicontinuity there is a first $\beta$ for which the ellipsoid has a point on its boundary such that $u=v$. For such a point $R=(s, y)$ we showed that $y=x_{1}$, thus $R \in \Delta$, which contradicts the fact that $u<v$ on $\Delta$.

We thus conclude that $u\left(t_{0}, x\right)=v\left(t_{0}, x\right)$ for all $x \in U$.

Now, we give a strict comparison principle on a region $[t, T[\times U$.

THEOREM 9 Assume that the coefficients of $\mathcal{L}^{t}$ are bounded and locally Lipschitz, and that $\psi$ is a continuous function. Let $U$ be a domain in $\mathbb{R}^{n}$. Let $u$ (resp. $v$ ) be a viscosity subsolution (resp. a 
viscosity supersolution) of

$$
\left\{\begin{array}{l}
\partial w / \partial t+\mathcal{L}^{t} w=0 \\
\forall x \in U, \quad w(T, x)=\psi(T, x)
\end{array}\right.
$$

such that $u(t, x) \leqslant v(t, x)$ for all $t \in[0, T]$ and $x \in U$. If there exists $\left.\left(t_{0}, x_{0}\right) \in\right] 0, T[\times U$ such that $u\left(t_{0}, x_{0}\right)=v\left(t_{0}, x_{0}\right)$, then $u(t, x)=v(t, x)$ for all $(t, x) \in\left[t_{0}, T[\times U\right.$.

We first show a lemma:

Lemma 10 Let $t_{0}>0$. If there exists $P_{0}=\left(x_{0}, t_{0}\right)$ such that $u\left(P_{0}\right)=v\left(P_{0}\right)$, then for every rectangle

$$
R_{0}=\left\{(t, x): t \in\left[t_{1}, t_{2}\right], x_{0}^{i}-a^{i} \leqslant x^{i} \leqslant x_{0}^{i}+a^{i}\right\},
$$

there exists $Q \in R_{0}$ with $Q \neq P_{0}$ and $u(Q)=v(Q)$.

Proof. Otherwise, we choose $R_{0}$ in which $u<v$. We let $h(t, x)=t_{0}-t+K\left\|x-x_{0}\right\|^{2}$, where $K$ is chosen such that $\mathcal{L}^{t} h\left(P_{0}\right)+(\partial h / \partial t)\left(P_{0}\right)>0$. By continuity, there exists $r>0$ such that $\mathcal{L}^{t} h+\partial h / \partial t>0$ on $\left.B\left(P_{0}, r\right) \subset\right] 0, T\left[\times U\right.$. Let $A=B\left(P_{0}, r\right) \cap\{(t, x): h(t, x)<0\}$. There are two parts of the boundary of $A$ : the first where $h=0$ and the second which is part of the sphere. $\partial A$ is a compact subset; so by lower semicontinuity, there exists $\epsilon>0$ such that $u \leqslant v+\epsilon h$ on $\partial A$. We then conclude with the same kind of argument as in the previous proposition: we get $u \leqslant v+\epsilon h$ on $A$ and derive a contradiction.

Corollary 11 If there exists $P_{0}=\left(x_{0}, t_{0}\right)$ with $t_{0}>0$ such that $u\left(P_{0}\right)=v\left(P_{0}\right)$, then $u=v$ for all $(t, x) \in\left[t_{0}, T\right] \times U$.

Proof. We first prove that there exists $P_{0}=\left(x_{0}, t_{0}\right)$ such that $u\left(P_{0}\right)=v\left(P_{0}\right)$, then $u=v$ on every rectangle

$$
R_{0}=\left\{(t, x): t \in\left[t_{0}, t_{2}\right], x_{0}^{i}-a^{i} \leqslant x^{i} \leqslant x_{0}^{i}+a^{i}\right\} .
$$

Let $R_{0}$ be such a rectangle with $u\left(P_{0}\right)=v\left(P_{0}\right)$ at $P_{0}=\left(\left(x_{1}+x_{2}\right) / 2, t_{1}\right)$. If we assume that $u-v$ is not constant in $R_{0}$, there exists $Q \in \bar{R}_{0}$ such that $u(Q)<v(Q)$. According to Proposition $8, Q$ does not belong to the plane $t=t_{1}$. On the segment $\left[P_{0}, Q\right]$ there is a first point $P_{1}$ from $Q$ such that $u\left(P_{1}\right)=v\left(P_{1}\right)$. We can assume $P_{1}=P_{0}$, otherwise we modify the rectangle. In the interior of the rectangle, we cannot have $u=v$; otherwise on the planes $t=$ const, we would have $u=v$ and thus on $\left.] P_{0}, Q\right]$, we would have a point such that $u=v$. If necessary we again make the rectangle smaller to have $u<v$ except on the plane $t=t_{1}$. This contradicts Lemma 10

REMARK 6 This strict comparison principle remains valid for any domain $U$. We will derive from it a local characterization similar to the one of Villeneuve [30] with much less restrictive hypotheses.

\subsection{Characterization of the exercise region}

Proposition 12 Assume that for every $t \in\left[0, T\left[, \mathcal{L}^{t} \psi\right.\right.$ is positive nonzero in the viscosity sense on a domain $U$ (i.e. $\psi$ is a viscosity subsolution of $\mathcal{L}^{t} w=0$ but is not a solution). Then

$$
\forall(t, x) \in[0, T[\times U, \quad u(t, x)>\psi(x) .
$$

In other words,

$$
\mathcal{E} \cap([0, T[\times U)=\emptyset
$$


Proof. Assume that there exists $\left(t_{0}, x_{0}\right) \in\left[0, T\left[\times U\right.\right.$ such that $u\left(t_{0}, x_{0}\right)=\psi\left(x_{0}\right)$. Recall that $u$, the solution of the obstacle problem, is a viscosity supersolution and $\psi$ a subsolution of $w_{t}+\mathcal{L}^{t} w=0$. According to Corollary 11 $u(t, x)=\psi(x)$ for all $t \in\left[t_{0}, T\right]$ and $x \in U$. However, if $u \leqslant w$ in ]$t_{0}, T[\times U$ with $w$ a test function, we can choose $w$ which does not depend on time because $u$ does not (we can choose $x \mapsto w(t, x)$ ). Then it is enough to notice that $u$ is the viscosity solution of

$$
\left\{\begin{array}{l}
\max \left(\psi-w, \partial w / \partial t+\mathcal{L}^{t} w\right)=0, \\
\forall x \in U, \quad w(T, x)=\psi(T, x),
\end{array}\right.
$$

and so $\mathcal{L}^{t} \psi=0$ in the viscosity sense. This is a contradiction.

REMARK 7 In particular, we derive the result for $U=\mathbb{R}^{n}$, i.e.,

$$
\mathcal{L}^{t} \psi>0 \text { in the viscosity sense for all } t \in[0, T[\Rightarrow \mathcal{E}=\emptyset .
$$

We next show the converse.

Proposition 13 If $\mathcal{E}=\emptyset$ then $\mathcal{L}^{T} \psi$ is a nonnegative measure on $\mathbb{R}^{n}$.

Proof. Let $\theta$ be a nonnegative function in $C_{0}^{\infty}\left(\mathbb{R}^{n}\right)$, the set of smooth functions with compact support. We know that $u \in C^{1,2}\left(\left[0, T\left[\times \mathbb{R}^{n}\right)\right.\right.$ because $\mathcal{E}=\emptyset$ and $u$ is a classical solution of $u_{t}+\mathcal{L}^{t} u=0$ on $\left[0, T\left[\times \mathbb{R}^{n}\right.\right.$. We also know from Proposition 1 that $u \in C^{0}\left([0, T] \times \mathbb{R}^{n}\right)$. According to Heine's theorem $u$ is uniformly continuous on $[0, T] \times \operatorname{supp} \theta$. Thus $u(t, \cdot) \rightarrow \psi(\cdot)$ uniformly on $\operatorname{supp} \theta$ when $t$ tends to $T$. Integrating the equation from $t<T$ to $T$, we get

$$
0 \leqslant \psi(x)-u(t, x)=\int_{t}^{T} \mathcal{L}^{s} u(s, x) \mathrm{d} s .
$$

Multiplying by $\theta$ and integrating with respect to $x$ yields

$$
\frac{1}{t-T} \int_{t}^{T} \int_{\operatorname{supp} \theta} \mathcal{L}^{s} u(s, x) \theta(x) \mathrm{d} x \mathrm{~d} s \leqslant 0 .
$$

One easily concludes using integration by parts and letting $t$ tend to $T$, the convergence being uniform.

Fianlly, we establish another localization criterion (shown in Villeneuve [30] in the case of constant coefficients). Consider the $t$-sections of the exercise region:

$$
\mathcal{E}_{t}=\{x \mid u(t, x)=\psi(x)\} .
$$

PROpOSITION 14 (1) $\mathcal{E} \subset\{(t, x): \psi(x)>0\}$

(2) $\mathcal{L}^{t} \psi \leqslant 0$ in the viscosity sense on $\bigcup_{t<T} \mathcal{E}_{t}$.

(3) In the homogeneous case, $\mathcal{L} \psi \geqslant 0$ in the viscosity sense on $\left(\overline{\bigcup_{t<T} \mathcal{E}_{t}}\right)^{c}$.

Proof. (1) holds because the price of the American option takes the 0 value only when $t=T$ according to the strict maximum principle.

(2) is a consequence of the viscosity PDE solved by $u$.

To prove (3), we notice that $u_{t}+\mathcal{L} u=0$ in the classical sense on $] 0, T\left[\times\left(\overline{\bigcup_{t<T} \mathcal{E}_{t}}\right)^{c}\right.$ and that $u_{t} \leqslant 0$. Therefore $\mathcal{L} u \geqslant 0$ and we can conclude as in the proof of Proposition 13 . 


\section{Influence of parameters on prices and convexity for one underlying asset}

In this section we investigate the influence of the volatility parameter on the price of an American option when its payoff is convex with respect to the price of the asset. In particular, we show by means of PDE technics that the price is nondecreasing with respect to the volatility parameter. The understanding of the influence of this parameter is very relevant from a market point of view. Volatility parameters are often obtained by calibrating prices and the price of options is given in terms of volatility.

In this section we only study one asset option. The price of this asset is assumed to solve

$$
\mathrm{d} s_{t}=s_{t} \mu(t) \mathrm{d} t+s_{t} \sigma\left(t, s_{t}\right) \mathrm{d} W_{t} .
$$

Here $\mu$ is the drift term which can typically take into account dividends paid by the asset. $\mu$ is assumed to be a Lipschitz function. The study includes the case of the American put with payoff $\psi(s)=(K-s)_{+}$, which is a convex function. The price of the American option solves the viscosity PDE

$$
\left\{\begin{array}{l}
\forall s \in \mathbb{R}^{+} \forall t \in[0, T], \quad \max \left(\psi-u, \frac{\partial u}{\partial t}+\frac{\sigma(t, s)^{2}}{2} s^{2} u_{s s}+\mu(t) s u_{s}-r(t) u\right)=0, \\
\forall s \in \mathbb{R}^{+}, \quad u(T, s)=\psi(s) .
\end{array}\right.
$$

We restate the comparison principle of Theorem 3 using the change of variables $x=\ln (s)$.

Proposition 15 Assume that the coefficients of $\mathcal{L}^{t}$ are bounded and locally Lipschitz, and that $\psi$ is a continuous function. Let $u$ (resp. $v$ ) be a subsolution (resp. a supersolution) of

$$
\left\{\begin{array}{l}
\max \left(\psi-w, \partial w / \partial t+\mathcal{L}^{t} w\right)=0 \\
\forall s \in \mathbb{R}_{+}, \quad w(T, s)=\theta(T, s)
\end{array}\right.
$$

such that $u(T, s) \leqslant v(T, s)$ for all $s \in \mathbb{R}_{+}^{*}$. Moreover, assume that there exist positive constants $C_{1}, C_{2}, \lambda$ such that for all $(t, s) \in[0, T] \times \mathbb{R}_{+}^{*}$,

$$
\left\{\begin{array}{l}
|u(t, s)| \leqslant C_{1} s^{\lambda}+C_{2} s^{-\lambda} \\
|v(t, s)| \leqslant C_{1} s^{\lambda}+C_{2} s^{-\lambda} \\
|\psi(s)| \leqslant C_{1} s^{\lambda}+C_{2} s^{-\lambda}
\end{array}\right.
$$

We now use a penalized problem to show the propagation of convexity. We can assume $\sigma, \mu$ and $r$ to be smooth enough, otherwise we use a smooth approximation.

Proposition 16 If $\psi$ is a convex function, then so is $u(t, \cdot)$ for every $t \in[0, T[$.

Proof. We introduce the penalized PDE which has a unique solution which converges to $u$. We assume that $\psi$ is bounded, otherwise we use a cut-off procedure. Consider the PDE

$$
\left\{\begin{array}{l}
\forall s \in \mathbb{R}^{+} \forall t \in\left[0, T\left[, \quad-\frac{\partial v}{\partial t}-\frac{\sigma^{2}}{2} s^{2} v_{s s}-\mu s v_{s}+r v-\frac{1}{\epsilon} \phi_{\epsilon}\left(\psi_{\epsilon}-v\right)=0,\right.\right. \\
\forall s \in \mathbb{R}^{+}, \quad v(T, s)=\psi_{\epsilon}(s),
\end{array}\right.
$$

where $\phi_{\epsilon}$ is a function twice continuously differentiable from $\mathbb{R}$ into $\mathbb{R}$ with polynomial growth together with its first and second derivatives; moreover $\phi_{\epsilon}(0)=0$. We also choose $\phi_{\epsilon}$ to be nondecreasing, convex and to converge to the function $x \mapsto x_{+}$pointwise when $\epsilon$ tends to 0 . The 
function $\psi_{\epsilon}$ is an approximation of $\psi$ which is convex, twice continuously differentiable from $\mathbb{R}$ into $\mathbb{R}$ with polynomial growth together with its first and second derivatives (a mollification fulfills these conditions). This PDE penalizes the solution being below $\psi$ when $\epsilon$ tends to 0 .

Under these conditions $\left(\mathrm{S}_{\epsilon}\right)$ has a unique solution $v_{\epsilon} \in C^{1,4}(] 0, T\left[\times \mathbb{R}^{+}\right)$with polynomial growth together with its first and second derivatives. The stability theory for viscosity solutions (see [11] for details) asserts that $v_{\epsilon}$ tends to $u$ uniformly on each compact subset.

To prove that $u$ is convex we first prove that $v_{\epsilon}$ is convex by differentiating $\left(\mathrm{S}_{\epsilon}\right)$ twice. This leads to a system solved by $w=\left(v_{\epsilon}\right)_{s s}$ :

$$
\left\{\begin{aligned}
\forall s \in \mathbb{R}^{+} \forall t \in & {\left[0, T\left[, \quad-\frac{\partial w}{\partial t}-\frac{\sigma^{2}}{2} s^{2} w_{s s}+\left(-2 \sigma \sigma^{\prime} s^{2}-2 s \sigma^{2}-\mu s\right) w_{s}\right.\right.} \\
& +\left(r-2 \mu-\sigma^{\prime 2} s^{2}-\sigma \sigma^{\prime \prime} s^{2}-4 \sigma \sigma^{\prime} s-\sigma^{2}+\frac{1}{\epsilon} \phi_{\epsilon}^{\prime}\left(\psi_{\epsilon}-v_{\epsilon}\right)\right) w \\
& =\frac{1}{\epsilon}\left(\left(\psi_{\epsilon}\right)_{s}-\left(v_{\epsilon}\right)_{s}\right)^{2} \phi_{\epsilon}^{\prime \prime}\left(\psi_{\epsilon}-v_{\epsilon}\right)+\frac{1}{\epsilon}\left(\psi_{\epsilon}\right)_{s s} \phi_{\epsilon}^{\prime}\left(\psi_{\epsilon}-v_{\epsilon}\right) \geqslant 0, \\
\forall s \in \mathbb{R}^{+}, \quad w(T, s)=\left(\psi_{\epsilon}\right)_{s s}(s) \geqslant 0 . &
\end{aligned}\right.
$$

The classical maximum principle for parabolic PDEs asserts that $w$ is nonnegative. Thus, $v_{\epsilon}$ is convex. The result is then obtained by letting $\epsilon$ tend to 0 .

We now study the behavior of the price when the volatility changes. We compare the price of two options with the same payoff but whose asset prices are not the same. We then generalize Touzi's results on the American put [28].

For $i=1,2$, the option whose price is $u_{i}$ corresponds to the diffusion

$$
\mathrm{d} s_{t}=s_{t} \mu(t) \mathrm{d} t+s_{t} \sigma_{i}\left(t, s_{t}\right) \mathrm{d} W_{t} .
$$

We now want to show that $u_{1} \leqslant u_{2}$ when $\sigma_{1} \leqslant \sigma_{2}$. To do this, we show that $u_{1}$ is a viscosity subsolution of the PDE solved by $u_{2}$.

PROPOSITION 17 The price of an American option on a convex payoff is nondecreasing with respect to the volatility parameter: $u_{2} \geqslant u_{1}$ pointwise if $\sigma_{2} \geqslant \sigma_{1}$ pointwise.

Proof. Let $\Phi$ be a test function such that $u_{1}-\Phi$ has a local maximum at $\left(t_{0}, x_{0}\right)$. By the definition of viscosity subsolutions,

$$
\left\{\begin{array}{l}
\max \left(\psi-\Phi, \partial \Phi / \partial t+\mathcal{L}_{1}^{t} \Phi\right)\left(t_{0}, x_{0}\right) \geqslant 0 \\
\Phi\left(t_{0}, x_{0}\right) \leqslant \psi\left(x_{0}\right) .
\end{array}\right.
$$

We next show that $u_{1}$ is a subsolution of the PDE corresponding to $\sigma_{2}$. In fact, it is sufficient to show that $\mathcal{L}_{1}^{t} \Phi\left(t_{0}, x_{0}\right) \leqslant \mathcal{L}_{2}^{t} \Phi\left(t_{0}, x_{0}\right)$. This is equivalent to

$$
\left(\sigma_{1}^{2}-\sigma_{2}^{2}\right) D_{x}^{2} \Phi\left(t_{0}, x_{0}\right) \leqslant 0 .
$$

By Lemma 3 in [10], since $u_{1}(t, \cdot)$ is convex and $u_{1}(t, \cdot)-\Phi(t, \cdot)$ has a local maximum at $x_{0}$, we get $D_{x}^{2} \Phi\left(t_{0}, x_{0}\right) \geqslant 0$. Finally, $\sigma_{1}^{2}-\sigma_{2}^{2} \leqslant 0$ and the result is shown.

\section{Examples}

We are going to show how our results can be applied to some standard cases like the American call or American put. It is well known that an American put on one underlying asset has a nonempty exercise region unlike an American call on one asset paying no dividend. 


\section{The American put}

We can give the shape of the exercise region for an American put even in a case where the volatility varies. To treat this example we work directly on the price of the asset and not on its logarithm (using the standard change of variable). The price process $\left(S_{t}\right)$ solves, under the risk-neutral probability,

$$
\mathrm{d} S_{t}=S_{t} \mu(t) \mathrm{d} t+S_{t} \sigma\left(t, S_{t}\right) \mathrm{d} W_{t},
$$

where $W_{t}$ is a standard Brownian motion and $\mu$ is the drift term which can take dividend into account.

Proposition 18 The exercise region of the American put with payoff $\psi(S)=(K-S)_{+}$is nonempty and there exists a function $\varphi$ with $\varphi(t)<K$ for $t<T$ such that the $t$-sections $\mathcal{E}_{t}$ are given by $\mathcal{E}_{t}=[0, \varphi(t)]$.

Proof. The fact that $0 \in \mathcal{E}_{t}$ comes from Remark 5 . Moreover, in Section 4, we showed that the put price is convex with respect to the asset price. Therefore, Proposition 16 implies that the $t$-sections are closed intervals included in $[0, K[$.

\section{The American call with stochastic volatility}

Let us examine the American call with a non-dividend-paying asset when the volatility is stochastic. We consider an asset whose $\log$ price process $X_{t}$ is described by the following bivariate SDE:

$$
\begin{aligned}
\mathrm{d} X_{t} & =r(t) \mathrm{d} t+\sigma\left(t, X_{t}, Y_{t}\right)\left(\sqrt{1-\rho^{2}\left(t, X_{t}, Y_{t}\right)} \mathrm{d} W_{t}^{1}+\rho\left(t, X_{t}, Y_{t}\right) \mathrm{d} W_{t}^{2}\right), \\
\mathrm{d} Y_{t} & =v\left(t, Y_{t}\right)+\gamma\left(t, Y_{t}\right) \mathrm{d} W_{t}^{2},
\end{aligned}
$$

where $W=\left\{W_{t}=\left(W_{t}^{1}, W_{t}^{2}\right), \mathcal{F}_{t}, 0 \leqslant t \leqslant T\right\}$ is a standard Brownian motion under the risk-neutral probability $P$, and $r$ is the instantaneous interest rate. This is an extension of the model introduced by Hull and White [16].

The payoff of an American call is then $\psi(x, y)=\left(e^{x}-K\right)_{+}$. To use our results, we assume that this option has two underlying assets but that the payoff depends only on the first asset.

We have $\mathcal{L}^{t} \psi(x, y)=r(t) K$ if $x>\ln (K)$, and $\mathcal{L}^{t} \psi=0$ if $x<\ln (K)$. Thus, to prove that $\mathcal{L}^{t} \psi$ is positive in the viscosity sense it suffices to examine its behavior around $a=\ln (K)$. In fact, it is not possible to find a function $w \in C^{2}$ such that $w(a)=0$ and $w \geqslant \psi$ in a neighborhood of $a=\ln (K)$ because the left derivative at $a$ would differ from the right derivative.

Thus, we can assert that for every $t \in\left[0, T\left[, \mathcal{L}^{t} \psi>0\right.\right.$ in the viscosity sense on $\mathbb{R}^{2}$. According to Proposition 12, we recover Merton's result [24] even when we have a stochastic volatility:

Proposition 19 It is never optimal to exercise the American call written on a non-dividendpaying asset before expiry. It coincides with the European call.

REMARK 8 Ishii [17] showed, under some reasonable hypotheses on the coefficients of the diffusion, that $\mathcal{L}^{t} \psi \geqslant 0$ in the viscosity sense on $\mathbb{R}^{n}$ is equivalent to $\mathcal{L}^{t} \psi \geqslant 0$ in the distribution sense on $\mathbb{R}^{n}$ when $\psi$ is a continuous function. We could have calculated only $\mathcal{L}^{t} \psi$ in the distribution sense but we wanted to illustrate the viscosity theory. 
The call on the minimum of two assets

We can study another example treated in Villeneuve [30]: the American call written on the minimum of two non-dividend-paying assets. Unlike the American call, its exercise region is not empty.

In order to study such examples, we use the Black-Scholes framework. The stochastic differential equation solved by the logarithms of prices is then

$$
\mathrm{d} X_{t}^{i}=\left(r(t)-\frac{1}{2} \sum_{j=1}^{2} \sigma_{i j}\left(t, X_{t}\right)^{2}\right) \mathrm{d} t+\sum_{j=1}^{2} \sigma_{i j}\left(t, X_{t}\right) \mathrm{d} W_{t}^{j}, \quad i=1,2,
$$

where $W=\left\{W_{t}=\left(W_{t}^{1}, W_{t}^{2}\right), \mathcal{F}_{t}, 0 \leqslant t \leqslant T\right\}$ is a standard Brownian motion under $P$, and $r$ is the instantaneous interest rate. We have the payoff function $\left.\psi(x)=\left(\min \left(e^{x_{1}}, e^{x_{2}}\right)-K\right)_{+}\right)$. We then calculate

$$
\mathcal{L}^{t} \psi=-\frac{\sqrt{2}}{2}\left(a_{11}-2 a_{12}+a_{22}\right) e^{x_{1}} \mathrm{~d} \lambda+r K \chi\left\{x_{1} \neq x_{2} \mid \min \left(x_{1}, x_{2}\right)>\ln (K)\right\},
$$

where $\sigma \sigma^{*} / 2=\left(a_{i j}\right), \mathrm{d} \lambda$ is the Lebesgue measure on the half-line of equation $x_{1}=x_{2}, x_{1}>\ln (K)$, and $v$ is the normal to this line.

Moreover this distribution is not positive for $t=T$. Indeed $a_{11}(T, x)-2 a_{12}(T, x)+a_{22}(T, x)$ $>0$ since $A$ is a positive definite matrix. Using Proposition 13 , we get $\mathcal{E} \neq \emptyset$.

Since $\psi$ is smooth on $\left\{x_{1}>x_{2}\right\} \cap\left\{x_{1} \neq \ln (K)\right\}$ and satisfies $\mathcal{L}^{t} \psi>0$ in the classical way, we proceed as in the one-dimensional case to show that for $t \in\left[0, T\left[, \mathcal{L}^{t} \psi>0\right.\right.$ in the viscosity sense on the domain $\left\{x_{1}>x_{2}\right\}$. The same holds on $\left\{x_{1}<x_{2}\right\}$. Using again Proposition 12, we conclude that $\mathcal{E}$ is included in $\left\{x_{1}=x_{2}\right\} \times[0, T[$.

\section{Basket options}

As a last example, we investigate a basket put option, that is, an option with payoff $\left(K-\sum_{i=1}^{n} e^{x_{i}}\right)_{+}$. It is one of the most often used contracts by practitioners. The logarithms of prices solve the following SDE:

$$
\mathrm{d} X_{t}^{i}=\left(r(t)-\delta_{i}\left(t, X_{t}\right)+\frac{1}{2} \sum_{j=1}^{n} \sigma_{i j}\left(t, X_{t}\right)^{2}\right) \mathrm{d} t+\sum_{j=1}^{n} \sigma_{i j}\left(t, X_{t}\right) \mathrm{d} W_{t}^{j}, \quad i=1, \ldots, n .
$$

The previous results show that the exercise region is nonempty and that

$$
\bigcup_{t<T} \mathcal{E}_{t} \subset\left\{\sum \delta_{i}(t, x) \exp \left(x_{i}\right) \leqslant r K, \sum \exp \left(x_{i}\right)<K\right\} .
$$

In the case of constant coefficients Villeneuve [30] proved

$$
\bigcup_{t<T} \mathcal{E}_{t}=\left\{\sum \delta_{i} \exp \left(x_{i}\right)<r K, \sum \exp \left(x_{i}\right)<K\right\}
$$

\section{Acknowledgements}

I would like to thank both of my Ph.D. advisors Henri Berestycki and Jérôme Busca. I also thank Daniel Gabay for his fruitful comments. 


\section{REFERENCES}

1. Alvarez, O., Lasry, J.-M., \& Lions, P.-L. Convex viscosity solutions and state constraints. J. Math. Pures Appl. 76 (1997), 265-288. Zbl 0890.49013 MR 1441987

2. BARDI, M. \& DA LiO, F. On the strong maximum principle for fully nonlinear degenerate elliptic equations. Arch. Math. (Basel) 73 (1999), 276-285. Zbl 0939.35038 MR 1710100

3. Barles, G. Solutions de Viscosité et Équations Elliptiques du Deuxième Ordre. Cours de l'Univ. de Tours (1997).

4. Bensoussan, A. \& Lions, J. L. Applications of Variational Inequalities in Stochastic Control. NorthHolland (1982). Zbl 0478.49002 MR 0653144

5. Bensouss an, A. On the theory of option pricing. Acta Appl. Math. 2 (1984), 139-158. MR 0748007 Zbl 0554.90019

6. Berestycki, H. Utilisation des EDP en finance. Cours de DEA de l'Univ. Paris 6.

7. Black, F. \& SCHOles, M. The pricing of corporate liabilities. J. Political Economy 81 (1973), 637-654. Zbl 01602446

8. Broadie, M. \& Detemple, J. The valuation of American options on multiple assets. Math. Finance 7 (1997), 241-286. Zbl 0882.90005 MR 1459060

9. CRandall, M. G. Semidifferentials, quadratic forms and fully nonlinear elliptic equations of second order. Ann. Inst. H. Poincaré Anal. Non Linéaire 6 (1989), 419-435. Zbl 0734.35033 MR 1035337

10. Crandall, M. G. \& Ishit, H. The maximum principle for semicontinuous functions. Differential Integral Equations 3 (1990), 1001-1014. Zbl 0723.35015 MR 1073054

11. Crandall, M. G., Ishit, H., \& LiOns P.-L. User's guide to viscosity solutions of second order partial differential equations. Bull. Amer. Math. Soc. 27 (1992), 1-67. Zbl 0755.35015 MR 1118699

12. El Karoui, N., Kapoudjian, C., Pardoux, E., Peng, S., \& Quenez, M. C. Reflected solutions of backward SDE's, and related obstacle problems for PDE's. Ann. Probab. 25 (1997), 702-737.

13. Friedman, A. Partial Differential Equations of Parabolic Type. Prentice-Hall (1964). Zbl 0144.34903 MR 0181836

14. Gilbarg, D. \& Trudinger, N. Elliptic Partial Differential Equations of Second Order. Springer (1977). Zbl 0361.35003 MR 0473443

15. Harrison, J. M. \& Pliska, S. R. Martingales and stochastic integrals in the theory of continuous trading. Stochastic Process. Appl. 11 (1981), 215-260. Zbl $0482.60097 \mid$ MR 0622165

16. Hull, J. \& White, A. The pricing of options on assets with stochastic volatilities. J. Finance 3 (1987), 281-300.

17. IsHII, H. On the equivalence of two notions of weak solutions, viscosity solutions and distribution solutions. Funkcial. Ekvac. 38 (1995), 101-120. Zbl 0833.35053 MR 1341739

18. Jaillet, P., LAmberton, D., \& Lapeyre, B. Variational inequalities and the pricing of American options. Acta Appl. Math. 21 (1990), 263-289. Zbl 0714.90004 MR 1096582

19. Kampen, J. On American derivatives and related obstacle problems. Int. J. Theor. Appl. Finance 6 (2003), 565-591. MR 1999825

20. Karatzas, I. On the pricing of American options. Appl. Math. Optim. 17 (1988), 37-60. Zbl 0699.90010 MR 0908938

21. Ladyzhenskaya, O., Solonnikov, V., \& Uraltseva, N. Linear and Quasilinear Elliptic Equations. Amer. Math. Soc. (1968). Zbl 0164.13002 MR 0244627

22. LiOns, P.-L. Optimal control of diffusion processes and Hamilton-Jacobi-Bellman equations. Parts I and II. Comm. Partial Differential Equations 8 (1983), 1101-1174, Zbl 0716.49022 MR 0709164; 8 (1983), 1229-1276. Zbl 0716.49023 MR 0709162

23. MCKeAn, H. P. A free boundary problem for the heat equation arising from a problem in mathematical economics. Industrial Management Rev. 6 (1965), 32-39. 
24. Merton, R. C. Theory of rational option pricing. Bell J. Econom. Management Sci. 4 (1973), 141-183. MR 0496534

25. Nirenberg, L. A strong maximum principle for parabolic equations. Comm. Pure Appl. Math. 6 (1953), 167-177. Zbl 0050.09601 MR 0055544

26. ØKSEndal, B. Stochastic Differential Equations. 5th ed., Springer (2000). MR 1619188

27. Rodrigues, J. F. Obstacle Problems in Mathematical Physics. North-Holland (1987). Zbl 0606.73017

28. TouZI, N. American options exercise boundary when the volatility changes randomly. Appl. Math. Optim. 39 (1999), 411-422. Zbl 0937.60039 MR 1675122

29. VAn Moerbeke, P. On optimal stopping and free boundary problems. Rocky Mountain J. Math. 4 (1974), 539-578. Zbl 0323.60051 MR 0381188

30. Villeneuve, S. Exercise regions of American options on several assets. Finance Stochast. 3 (1999), 295-322. Zbl 1047.91037

31. Villeneuve, S. Options américaines dans un modèle de Black-Scholes multidimensionnel. Phd thesis, Univ. de Marne la Vallée (1999). 